\title{
Weight and consequences of post-marketing (-approval) experience
}

\section{G Kreutz}

Ann Rheum Dis 2002;61(Suppl II):ii90-ii91

1 ne European Union the term pharmacovigilance is normally used to describe post-marketing or, more precisely, post-approval experience after the regulatory approval of drugs and their introduction to the market under defined conditions. The term describes activities used to gather information on the effects of medicinal products, with emphasis on those aspects which have not, or could not have been, detected in the pre-approval phase of the development of the product.

The term was first introduced in France more than two decades ago when authorities there set up and developed a national system to determine possible adverse reactions caused by medicinal products and to confirm or reject these reactions. In addition to this pharmacovigilance system, nationally agreed and implemented methods for collecting, reporting, and imputing suspected adverse drug reactions have been used.

When the term was included in the European regulations for medicines its meaning was extended to include all regulatory aspects of the evaluation of proprietary medicinal products resulting from, and relevant to, the description of the regulatory status and use of medicinal products after post-marketing authorisation of the product in the Union or in any of its member states.

Pharmacovigilance is necessary for many reasons and provides important information about the safe use of medicines.

It should be recognised that at the time of approval of a drug there are major limitations to knowledge about all those drugs which contain new active ingredients or possess other features relevant to their clinical effects. The patient group included in a pre-approval clinical trial is normally highly selected, owing to requirements related to the methodology of the trial, and is not, or only to a certain extent, representative of the population which will subsequently use the drug. Thus the information obtained will be incomplete and will not apply to all circumstances, contributing to the individual responses to exposure to a drug. Age and many constitutional factors, comorbidity, comedication are among those factors of major importance to an individual person's response. Other factors are limited numbers of exposed patients and limited duration of exposure. Targeted approaches to the detection, evaluation, and follow up of specific risks, and targeted approaches to determine the frequency of risks or their underlying mechanisms are often missing or incompletely considered.

Such limited knowledge at the time of approval means among other things that there are uncertainties about the frequencies of rare adverse reactions and almost no information about very rare or less frequent adverse reactions. Long term exposure effects on therapeutic or adverse effects are limited or non-existent. Mechanisms underlying adverse effects or predisposing conditions in patients are either studied only to the extent required by the clinical trial protocol or are lacking because insufficient consideration was given to the direction in which studies might have been planned and organised. Prevention of specific adverse reactions caused by the use of a medicinal product has normally only been studied to a limited extent, and most adverse reactions have not even been identified or could not possibly have been identified.
The purpose of pharmacovigilance as described in the European regulations is given in the Notice to Marketing Authorisation Holders Pharmacovigilance Guidelines dated 29 January 1999 (CPMP/PhvWP/108/99):

"Pharmacovigilance activities come within the scope of quality, safety, and efficacy, as new information is accumulated of the medicinal product under normal conditions of use in the marketing situation. Pharmacovigilance obligations apply to all authorised medicinal products, including those before 1 January 1995."

Obligations related to the fulfilment of this purpose are described for all the relevant stakeholders or responsible bodies.

- Marketing authorisation holders:

- "The marketing authorisation holders need to ensure that they have an appropriate system of pharmacovigilance in place in order to assure responsibility for their product on the market and to ensure that appropriate action be taken, when necessary. This includes.............at least one appropriately qualified person responsible for pharmacovigilance............., and the establishment of a system for the collection, preparation and submission of expedited adverse drug reactions and periodic safety update reports to competent authorities."

- Competent authorities (member states):

- "The authorities of the member states are the competent authorities for products authorised nationally through national procedures, including their mutual recognition procedure. The responsibilities for pharmacovigilance rest with the competent authorities of all the member states in which the authorisations are held."

- Competent authorities (European Commission):

- "The European Commission is the competent authority and responsible for decisions on the basis of CPMP opinions related to centrally authorised products and those products subject to the procedures of Articles 13 and 14 of Council Directive 75/319/EEC as amended."

System requirements for marketing authorisation holders are described in an additional more technical guideline (CPMP/PhvWP/1618/01) of 15 November 2001. According to this regulation the system needs to cover as its most important features expedited reporting, periodic safety update reporting, responding to requests for information from competent authorities, handling of urgent safety restrictions and safety variations, continuous monitoring of the safety profile of the authorised medicinal products, and notifying competent authorities and health professionals of any changes to benefit and risk. Additional and more general requirements are for the centralised procedure to meet CPMP requirements and to have in place internal audit for the pharmacovigilance system.

In conclusion, pharmacovigilance as described in European regulations for medicinal products is an important constituent of overall regulation of proprietary medicinal products. An efficient system provides new and important information 
about the effects and safe use of medicines. Pharmacovigilance is a responsibility shared between marketing authorisation holders, health professionals, and competent regulatory authorities. These responsibilities are distributed according to legal requirements and their ability to contribute. Procedures have to be implemented accordingly and be continuously checked for their efficiency and state of the art implementation. Responsible, experienced, and dedicated people are the cornerstones of effective post-approval handling of pharmaceutical products.

Author's affiliation

G Kreutz, Bundesinstitut für Arzneimittel und Medizinprodukte,

Department of Clinical Pharmacology Devices, Friedrich-Ebert-Allee 38 D-53113 Bonn, Germany 